\section{Case Management als Funktion eines Behandlungsverbundes}

Zusammenfassung: Case Management und Versorgungsstrukturen hängen miteinander zusammen. Sie können sich gegenseitig optimieren, wenn die Methodik von Case Management in die Verbundstruktur bis hinein in jedes Behandlungsmodul Eingang findet. In diesem Sinne wird Case Management eine Funktion eines Versorgungsverbundes. Ein Verbund definiert sich durch eine stringente Kooperation und die Optimierung von Case Management. Am Beispiel eines Therapieverbundes für Drogenabhängige werden Standards qualifizierter Kooperation definiert und der Modulcharakter beschrieben, den die einzelnen Behandlungsangebote erhalten. Steuerungsfunktion und Kontaktverantwortung müssen eindeutig an eine Person gebunden sein, die den "Gesamtbehandlungsplan“ vom Eintritt ins Hilfesystem bis hin zum Austritt ins Auge fasst. Ziel sollte es sein, systemübergreifend z. B. zwischen Jugend- und Drogenhilfe zu lotsen. Der vorgestellte Therapieverbund kann nur ein Anfang sein.

Schlüsselwörter: Case Management - Verbund - qualifizierte Kooperation - Steuerungsfunktion - Kontaktverantwortung - systemübergreifendes Case Management

Case Management as a Function in Care Services: Case Management and the structure of care services depend from each other. They could be optimized if the approach of case management was implemented. Therefore case management can be described as a function of an integrated system. An integrated system is defined by a qualified co-operation and a stringent integration and implementation of case management. The standards of qualified co-operation are defined and the open communicative character of the treatment services are described. The function of piloting and the responsibility for contacting has to be fixed to one person controlling the whole planning of treatment from entering the system to the exit. The problem of piloting between two different systems - for example drug services and juvenile help services and administration - is discussed.

Key words: Case Management - Integrated Systems Qualified Co-Operation - Piloting - Responsibility for Contacting - Piloting between Different Systems

Suchttherapie 2001; 2: 80-83

(c) Georg Thieme Verlag Stuttgart · New York ISSN 1439-9903
Case Management wird als aktive sozialpädagogische Vorgehensweise und Methode verstanden, die KlientInnen an Ressourcen des Hilfesystems heranführt, diese vermittelt und planvoll koordiniert, oder anders ausgedrückt, einen Versorgungszusammenhang erarbeitet. Zentrale Aufgaben sind dabei: bedarfsorientierte Hilfeplanung, Koordination und Steuerung des Gesamtprozesses. Ein planvolles Geleiten durch die Landschaft der Hilfesysteme verlangt nicht nur methodisches Rüstzeug, sondern Koordination und qualifizierte Zusammenarbeit der verschiedenen Versorgungssegmente und Behandlungsangebote. Definiert und qualifiziert ein regionaler Behandlungs- oder Versorgungszusammenhang seine Zusammenarbeit, sprechen wir von einem Verbund. Es ist Ziel eines Verbundes, eine personenzentrierte Hilfeplanung und Hilfeleistung in einem sinnvollen Gesamtkonzept $\mathrm{zu}$ erbringen. Zentrale Zielsetzung ist also Case Management. Wie ein Versorgungs- oder Behandlungsverbund eine neue Qualität von Case Management ermöglicht und umgekehrt eine Integration von Case Management die Behandlungsangebote selbst produktiv verändert, ist Gegenstand dieses Beitrags. Der Beitrag beruht auf den Erfahrungen eines Therapieverbundes für Drogenabhängige.

\section{Was ist ein Therapieverbund?}

Unter ökonomischem Druck des Wirtschaftsförderungsgesetzes (September 1996) öffneten sich Leistungsträger und Leistungsanbieter in der Suchtrehabilitation neuen Behandlungsideen und Formen der regionalen Kooperation. Eine Ausdifferenzierung der Rehabilitation Drogenabhängiger wurde gefördert (Langzeit-, Mittelzeit- und Kurzzeittherapie) bis hin zu neuen Behandlungsformen bei Drogenabhängigen wie Tagesklinik und ambulante Therapie. Aus heutiger Sicht markiert dieser Zeitpunkt ein Umdenken in Richtung auf eine integrierte ambulant-orientierte Versorgungsstruktur in der Suchttherapie und Rehabilitation.

Eine ambulant-orientierte Suchtbehandlung aber verlangt eine regionale Ausrichtung, die Entwicklung von qualifizierten Formen der Kooperation und Ansätze für eine integrierte stationär-ambulant-teilstationäre Behandlung. Diese Umorientierung setzte daher die Bildung regionaler Therapieverbünde auf die Tagesordnung.

Ein Therapieverbund definiert sich durch ein differenziertes regionales Versorgungs- und Behandlungsangebot und eine verbindliche qualifizierte Zusammenarbeit mit der Zielsetzung, die Versorgung und Behandlung Suchtkranker in einer 
Region zu verbessern. Einen solchen Therapieverbund möchte ich kurz vorstellen.

\section{Der Therapieverbund im Verein für Jugendhilfe e. V.}

Der Therapieverbund im Verein für Jugendhilfe umfasst vier Jugend- und Drogenberatungsstellen und die Rehabilitationseinrichtung Four Steps mit einem ausdifferenzierten Konzept aus Langzeit-, Mittelzeit- und Kurztherapie, Tagesklinik und Fachambulanz.

Dieser Verbund ermöglicht eine integrierte Rehabilitation von Drogenabhängigen, indem ambulante, tagesklinische und stationäre Angebote nahtlos und planvoll kombiniert werden - ein Schritt hin zu einer personenkonzentrierten Gesamtbehandlung. Grundlage für diese integrierte Rehabilitation war ein Rahmenkonzept, das zu Beginn des Jahres 97 erarbeitet wurde. Es verschränkt ambulante und stationäre Behandlung und wurde mit der LVA-Württemberg und der BfA verhandelt.

\section{Die Entwicklung einer modularen Versorgungs- und Behandungsstruktur}

Heute werden alle Behandlungseinheiten als „Module“, d.h. kombinierbar innerhalb eines Behandlungsverbundes, betrachtet, um wirklich individuelle, von den Betroffenen mitgestaltete, auf ihre Fähigkeiten und Ressourcen wie auf ihren individuellen Behandlungsbedarf zugeschnittene Behandlungswege möglich $\mathrm{zu}$ machen. Behandlungsmodule sind durch unterschiedliche Setting-Bedingungen, Zielsetzung und Inhalte definiert: stationäre Intensivbehandlung (Langzeit, Mittelzeit, Kurzzeit, Adaption), Tagesklinik, ambulante Reha und NachsorgeWG. Das folgende Schema beschreibt die möglichen kombinierten Behandlungswege im Therapieverbund.

\section{Behandlungswege}

Behandlungsangebote sind nicht per se „Behandlungsmodule“. Sie werden zu solchen, wenn sie „anschlussfähig“ werden, d.h., wenn sie sich mit anderen Modulen zu einem Ganzen verbinden lassen und eine effektive Kooperation mit dem umliegenden Versorgungssystem gewährleisten. Modulcharakter erhalten also Behandlungsangebote, die folgende Eigenschaften haben:

- sie haben eine offene, kooperative Struktur seitens der Mitarbeiter;

- sie sind fähig zur flexiblen Gestaltung der Behandlung (Zeit, Baustein);

- sie definieren sich als Teil eines Gesamtbehandlungsplans und können auftragsbezogen arbeiten;

- sie ordnen sich einer vorab vereinbarten Behandlungsplanung unter, die sie aktiv mitgestalten und fortschreiben;

- sie beziehen sich auf die externe Prozesssteuerung und -verantwortung bei der Fortschreibung der Rehaplanung und Beendigung;

- sie beteiligen sich aktiv, Strukturverantwortung (bezüglich der Kooperationsvereinbarung) sowohl intern wie für das Gesamt des Verbundes zu übernehmen.

\section{Case Management ist eine Funktion qualifizierter Kooperation im Therapieverbund}

Eine integrierte Suchtbehandlung verlangt, dass die Drogenhilfe verschiedene Professionen und Institutionen so koordiniert, dass daraus eine einheitliche Rehabilitation entsteht. D.h., das Behandlungssystem muss eine hinreichende Qualität der Koordination entwickeln:

- klare Definition der Kontakt- und Steuerungsverantwortung

- maximale personale Kontinuität

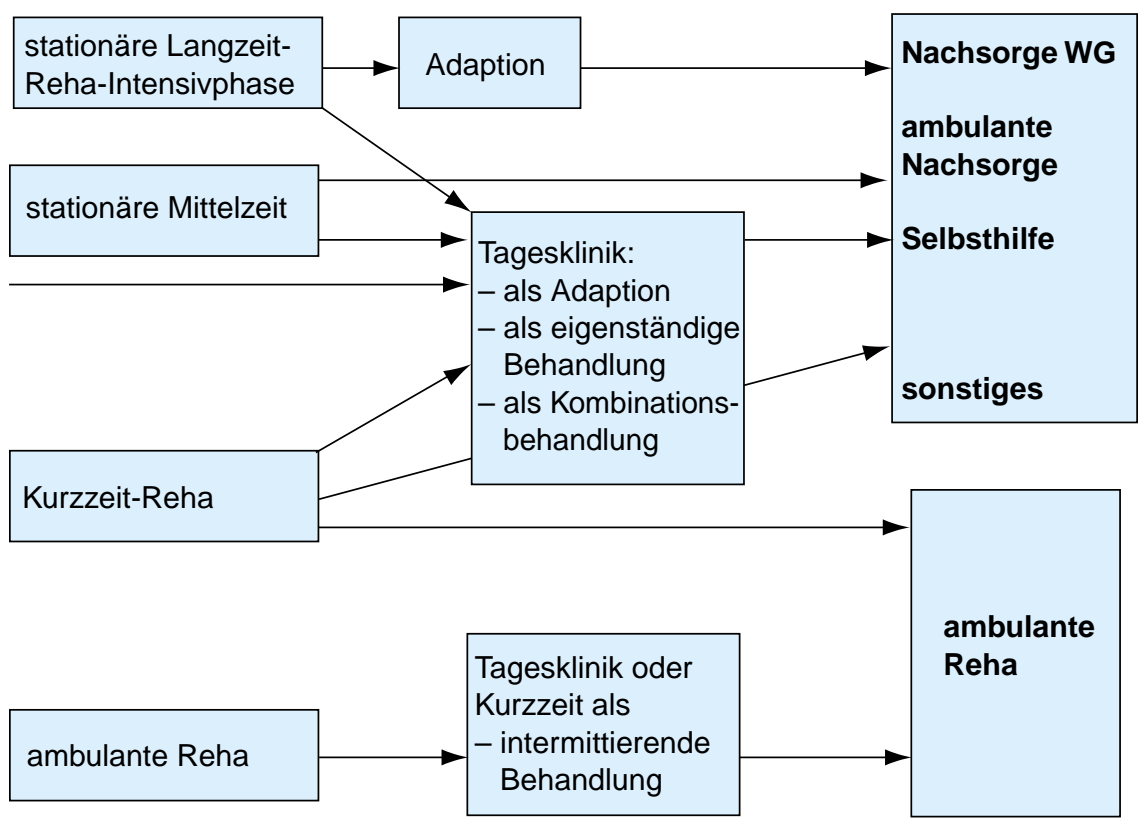

Abb. 1 Behandlungswege 
- durchgängige, einheitliche Rehaplanung

- klare Koordination im gesamten Behandlungsverlauf inkl. bei „Störungen“

- Konfliktmanagement unter den Beteiligten

- gemeinsame Fallbesprechung und Reflexion der Kooperation

- gemeinsame Konzeptionsentwicklung

Solche komplexen Anforderungen an Case Management verlangen feste, geregelte und überwachte Kooperationsstrukturen. Im Einzelnen:

- gemeinsame Diagnose und Indikationsstellung von Berater, Reha-Einrichtung, Rehabilitand und Leistungsträger. Besonders wichtig ist, dass der Rehabilitand sich mit seinen konkreten Behandlungsoptionen auseinandersetzen kann und zustimmt (siehe auch unten);

- Übergabe der Klienten, schriftliche Aufgabenstellung an das initiale Behandlungsmodul, die auch dem Rehabilitand vertraut ist. Weitergabe der schriftlichen internen Therapieplanung an den/die zuständigen KollegInnen, rasche Kontaktaufnahme zwischen Berater und Rehatherapeut;

- Kontakthalten während des ersten Reha-Abschnitts, vor allem bei Krisen, Entscheidungen um Entlassung, Planung der Weiterbehandlung und Fragen der künftigen Lebensgestaltung: telefonisch zwischen den Professionellen, durch obligatorischen Besuch der KlientInnen beim Berater im Rahmen von Heimfahrten, evtl. Besuch des Beraters vor Ort, Einleiten und Besuch ambulanter Weiterbehandlung bereits während der Endphase der Erstmoduls;

- bei Modulwechsel sind 3er-Gespräche (KlientIn, bisheriger und künftig zuständiger KollegeIn) hilfreich;

- eine Veränderung einer vorab geplanten Kombinationsbehandlung darf nur unter Hinzuziehen des Steuerungsverantwortlichen in Gang gesetzt werden;

- Übergabe und Fortschreibung der Rehaplanung, unmittelbare Weitergabe des Abschlussberichts;

- Rückmeldung über weiteren Verlauf und Reflexion nach Abschluss der Gesamtbehandlung;

- gemeinsame Intervision wechselnd bei allen Beteiligten (zwei- bis dreimal im Jahr): Fallbesprechung;

- gegenseitige Beteiligung bei konzeptionellen Überlegungen und Reflexion der Angebote (Angebotsmitgestaltung) z.B. durch gemeinsame Teamtage, Arbeitsgruppen, gezielte Teambesuche etc.;

- Überschaubarkeit und Transparenz: sich persönlich kennen, persönlich erreichen können, mit Entscheidungswegen vertraut sein; wesentliche Informationen mit Wissen des Klienten weitergeben können;

- Mechanismus etablieren, um Störungen unter Einbeziehen der Leitungsebene rasch zu klären;

- mittelfristig müssen auch Grundlagen einer gemeinsamen Ergebnisdokumentation und Qualitätsentwicklung geschaffen werden.

Dieser Auflistung lässt sich entnehmen, dass grundlegende Methoden von Case-Management in die Arbeitsweise der Behandlungsmodule Eingang finden und durch die Kooperationsvereinbarung institutionalisiert werden: Vereinbarungen mit dem Klienten über Zusammenarbeit, Assessment, Zielvereinbarung und Hilfeplanung, Verbindung des Klienten mit den Hilferessourcen, Monitoring und Ergebnisbewertung. Jedes Behandlungsmodul übernimmt solche Aufgaben und schreibt sie fort.
Das heißt Case Management und Versorgungs- und Behandlungsstrukturen hängen miteinander zusammen und können sich gegenseitig optimieren. Die spezifische Methodik von Case Management findet Eingang in die Verbundstruktur bis hinein in jedes Behandlungsmodul und seine Arbeitsweise. In diesem Sinne wird Case Management zu einer Funktion des Versorgungsverbundes und optimiert.

\section{Die Lotsenfunktion der Drogenberatung}

Die Lotsenfunktion ist verknüpft mit der Kontaktverantwortung. Sie liegt in unserem Verbund bei der vermittelnden Suchtberatungsstelle und wechselt nur, wenn der Klient ein neues Lebenszentrum begründet. Aufgabe ist es, einen koordinierten Gesamtverlauf und Schritte der Weiter-, Zwischenbehandlung oder Begleitung/ Beratung zu gewährleisten. Die Kontaktverantwortung wird wichtig bei „Störungen“ und Krisen der geplanten Gesamtbehandlung, vor allem bei vorzeitiger Behandlungsbeendigung. Die KlientInnen haben eine klare Anlaufstelle, und das Hilfesystem kann koordinierter reagieren. Es lernt, ruhig und geduldig mit „chaotischen Entwicklungen" umzugehen und die Betroffenen in angemesser Weise in Kontakt mit dem Hilfesysteme zu halten.

Die Ausübung dieser Funktion verlangt an Fähigkeiten:

- Aufrecht-erhalten-können einer an der Aufgabe orientierten, ungestörten Arbeitsbeziehung zu KlientInnen

- Genaue und persönliche Kenntnis der Behandlungsmodule und Angebote der Grundversorgung

- Aktiv praktische Hilfe gewähren, um Übergänge zu bewältigen

Strukturelle Voraussetzung ist: Bei Änderungen und Fortschreibung der Rehaplanung wird der Case-Manager vorab hinzugezogen; er hat gegenüber dem Behandlungsmodul das letzte Wort.

Das folgende Beispiel skizziert einen längeren Behandlungsverlauf, der im Behandlungsverbund über mehrere Stationen kooperativ gehandhabt wurde.

\section{Fallvignette}

Beginn der Beratung in Beratungsstelle 10/98

Herr F. hatte zuvor eine stationäre und im Anschluss ambulante Behandlung im Ausland ergebnislos durchlaufen. Diagnose: Störung durch multiplen Substanzgebrauch.

- 5/99: Vorbereitung auf stationäre Therapie (Einzel und Gruppe)

- 6/99: Beantragung Langzeitbehandlung

- 7/99: Aufnahme in der stationären Langzeittherapie

- 12/99: vorzeitig mit ärztlichem Einverständnis entlassen.

Nachdem im stationären Gruppensetting z.T. extreme Probleme mit Herrn F. aufgetreten waren (Konflikte im Zusammenspiel mit der Therapiegruppe, Schwierigkeiten, den relativ engen Rahmen auszuhalten), erfolgte Rücküberweisung in die Beratungsstelle, da Wunsch und Bereitschaft zur weiteren Mitwirkung an therapeutischen Maßnahmen gegeben waren.

Nach gegenseitiger Konsultation und diagnostischer Abklärung: 
- 1/00: Kostenantrag Kombinationstherapie teilstationär/ ambulant

- 2/00: Aufnahme in teilstationärer Therapie

Während der teilstationären Therapie finden zwischen Ambulanz und teilstationärer Einrichtung regelmäßige Konsultationen statt.

- 7/00: In Konsultationen und einem Übergabegespäch wird geklärt, dass Herr F. die ambulante Gruppenbehandlung vorläufig nicht in Anspruch nehmen wird, sondern Beratungsgespräche nach Bedarf. Trotz Bedenken stimmen teilstationäre Therapie und Ambulanz dieser Vorgehensweise als aussichtsreich zu.

Aktueller Stand: Bis dato ist Herr F. clean bei Drogen. Geringfügig trinkt er Alkohol. Den Kontakt zur Beratungsstelle hält er wie vereinbart. Aktuell bezieht er eine eigene Wohnung. Beim Arbeitsamt wird aktuell geklärt, ob Voraussetzungen zur Umschulung gegeben sind.

Voraussetzung für ein solches langfristiges Case Management war, dass Steuerungs- und Kontaktverantwortung klar definiert bei einem Mitarbeiter der Drogenberatung lagen. Jeder Schritt seitens des Hilfesystems wurde von hier aus koordiniert oder initiiert. Alle beteiligten Behandlungsmodule ordneten sich unter und verhielten sich gegenüber dem Klienten entsprechend der abgesprochenen Struktur.

\section{Case Management und Selbstmanagement}

Der Ausstiegsprozess aus Drogenabhängigkeit umfasst - wenn er gut verläuft - eine Zeit von 3 bis 5 Jahren und besteht aus Phasen von Selbstmanagement und von professioneller Beratung und Behandlung. Rehabilitation kann darin nur ein kurzer Zeitausschnitt sein, in der eine Entwicklung angestoßen wird, die in der Regie des Rehabilitanden über eine viel längere Zeit eigenverantwortlich weiterverfolgt werden wird.

Wir können also unmöglich die gesamte Ausstiegszeit professionell begleiten. Die Frage ist: Über welchen Zeitraum braucht unser Klient welche Form der Behandlung und der Hilfe? Geht er die Behandlungsschritte mit? Sind sie für ihn plausibel? Stimmt seine Planung mit derjenigen der Helfer überein? Sieht er sich unterstützt?

Entscheidend ist, den Rehabilitanden in allen Phasen der Behandlungsplanung bzw. Fortschreibung und die Voraussetzungen für jedes Behandlungsmodul gemeinsam zu überprüfen. Diese Qualität in der Organisation der Behandlungsmodule ermöglicht, dass KlientInnen den Ausstiegsprozess konstruktiv mitgestalten. Der Beratungs- und Behandlungsprozess muss unsere KlientInnen Schritt für Schritt befähigen und instandsetzen, diese Funktion produktiv zu übernehmen. Selbstmanagement und Case Management gehören eng zusammen und sind beide eine Funktion eines koordinierten modularen offenen Hilfesystems. Sie ergibt sich nicht so sehr aus der Fähigkeit des einzelnen Beraters/Therapeuten, sondern aus dem Zusammenwirken aller Beratungs- und Behandlungsmodule.

\section{Koordination zweier Hilfesysteme: Jugend- und Drogenhilfe}

Zum Verein für Jugendhilfe gehören auch Jugendhilfeeinrichtungen. Sie sind in diesen Verbund vielfältig einbezogen: in Kooperation von mobiler Jugendarbeit und Streetwork („Tandemteam“), Tages- bzw. Wohngruppenbetreuung und Drogenberatung bis hin zu intensivbetreuenden Maßnahmen für drogenabhängige Jugendliche, aber auch bezüglich Suchtbehandlung in Kooperation mit den Rehabilitationsangeboten: „Tandembehandlung“ zwischen Tagesklinik und Jugendhilfebetreuung. Hier arbeiten Betreuung der Jugendhilfe und Drogenbehandlung gleichzeitig, parallel und koordiniert zusammen. Dies ermöglicht es, Beziehungs- und Betreuungsbrüche bei drogenkonsumierenden Jugendlichen zu vermeiden und Suchtbehandlung effektiver einzubeziehen.

Für die Koordination gelten die schon geschilderten Regeln. Von besonderer Bedeutung ist: Wer übernimmt die Steuerungsfunktion zwischen beiden Hilfesystemen? Wer entscheidet z. B. über den weiteren Verlauf der Suchtbehandlung, wenn ein Konflikt zu bisherigen Planungen entsteht? Die Beantwortung dieser Frage war ein wichtiger Lernprozess für eine wirkungsvolle Koordination zwischen beiden Systemen: Die Steuerungs- und Kontaktfunktion liegt bei der Jugendhilfe. Der Entscheidungsträger der Jugendhilfemaßnahme sowie der Enscheidungsträger für die Jugendhilfeeinrichtung müssen einbezogen werden. Letztendlich wird die Entscheidung im Rahmen von Hilfeplangesprächen nach dem KJHG getroffen.

\section{Perspektiven von Case Management}

Sucht- und Drogenabhängigkeit ist eine komplexe Erkrankung, der wir eine ebenso vielfältige koordinierte Behandlung entgegensetzen müssen. Die Optimierung von Case Management innerhalb eines Rehabilitationsverbundes kann nur ein erster Schritt sein. Aus dem Blickwinkel einer Gesamtbehandlung verlangt eine wirkliche Sucht- und Drogenbehandlung die Koordination einer Vielzahl von Professionen und verschiedener Hilfesysteme. Es sollte möglich sein, vom Moment des Eintritts in das System der Drogenhilfe bis hin zum Verlassen desselben nach einem koordinierten Leitplan zu agieren. Da Suchtbehandlung nicht allein innerhalb des Drogenhilfesystems geleistet wird, schließt ein solches Case Management auch die Koordination verschiedener anderer Hilfemaßnahmen und Dienste ein (Akutversorgung, Substitution, Entgiftung, psyschiatrische Versorgung etc. wie auch das Justizsystem).

Dieser heute noch hypothetische Gedanke führt die Aufgabenstellung vor Augen, die auf die Drogenhilfe wartet. Sie müsste eine solche Qualität der Zusammenarbeit entwickeln und Case-Management-Methoden so weitgehend implementieren, dass es von außen gesehen genau den Anschein eines von Beginn an zielgerichteten Behandelns erweckt.

Rainer Baudis (Dipl.-Psych. Psychotherapeut)

Rehabilitationseinrichtung Four Steps Schorndorf Grabenstraße 28

73614 Schorndorf 